\title{
Covariance Control of Turbomachinery Vibrations
}

\author{
ElSayed M. ElBeheiry ${ }^{\dagger}$ \\ Department of Production Engineering and Mechanical Design, \\ Faculty of Engineering, Menoufia University, Shebin ElKom, Egypt
}

(Received 22 September 2000; accepted 6 March 2001)

\begin{abstract}
Two contrasting controller designs are proposed and investigated in this study. The goal is to suppress random vibrations of a high speed, flexible rotor-bearing system undergoing random base excitation. This turborotor is supported by two hydrodynamically lubricated fluid film bearings and carries three non-identical, heavy disks. It has been modelled by a Finite Element (FE) method that accounts for gyroscopic moments, rotary inertia, internal viscous and hysteretic damping and shear deformations. The application of the Extended Modal Reduction (EMR) method, that leaves the retained eigenfrequencies and mode shapes unaltered from their original values, provided a significant model truncation for reduced-order controller design. The first controller applies the active control forces directly to the rotor while the second one introduces these forces to either the bearing housing or the pedestal mass. The base excitations are modelled as stationary and Gaussian random processes in both the vertical and the horizontal direction. The two controllers are designed by using the State Covariance Assignment (SCA) theory that allows the achievement of prescribed variances by state or output feedback control. The results show that the two controllers marginally reduce vibrations due to random base excitations, when compared to the uncontrolled system.
\end{abstract}

${ }^{\dagger}$ Member of the International Institute of Acoustics and Vibration (IIAV)

\section{INTRODUCTION}

Active vibration control has become one of the top priorities of the vibration research community for many engineering applications. ${ }^{1-3}$ In the field of rotor dynamics, extensive research has been devoted mainly to producing rotor-bearing systems which operates at higher speeds and lighter weights and which possesses balanced, reliable operation. So many analysis procedures and control designs have been investigated theoretically and experimentally for this purpose. Analytical modelling methods, which describe the rotor-bearing dynamics by partial differential equations, are not as popular as discretisation approaches. This is why the finite element and transfer matrix methods have been among the most popular methods used for modelling rotor-bearing systems.

Spatial discretisation of rotor-bearing systems by FE methods usually leads to models with large numbers of elements and, consequently, larger numbers of degrees of freedom (DOF). The response and stability analyses of these models can be based sufficiently on reduced models obtained by modal or modified Guyan reduction techniques. This leads to simpler evaluations and makes substantial savings in computation time.

Although base-excited rotor-bearing-support systems commonly exist in practice, not so much attention have been paid to them as to some others. They normally exist onboard moving ground vehicles, ships and aircraft. In addition, rotating machines which operate during and just after earthquakes provide another important example of such systems. In such transient circumstances, these systems are supposed to continue to function properly. Otherwise, many additional emergencies are likely to take place in the region of earthquakes.
However, rotor-bearing systems undergoing seismic excitations are not amenable for analysis as are unbalanced systems. Seismic excitation is difficult to predict since it is not immediately known in what form and at what location an earthquake will occur. One can say that in regions having high seismological activity, very large gyroscopic forces are expected, while in regions having low or even moderate seismological activity the excitations can be considered as random inputs having different amplitudes at the rotor supports.

In practice we can assume that the majority of base excitations are random in nature. Rotors onboard running vehicles require the analysis of steady-state response due to persistent random excitations; while rotors installed in regions of low or moderate seismological activity necessitate transient response analysis. The performance degradation of fluid film bearings and magnetic bearings resulting from stochastic excitations makes it of vital importance to design controllers capable of dealing with these disturbances. Base excitations, if neglected, may make the rotor come into contact with the stator owing to the low stiffness of the magnetic or journal bearings.

Among the contributors to the analysis of rotor-bearingsupport systems subjected to random excitation are Lund ${ }^{4}$, Tessarzik et al. ${ }^{5,6}$, Asmis ${ }^{7}$, Subbaih et al. ${ }^{8}$, Gaganis et al. ${ }^{9}$, Soni and Srinvasen ${ }^{\mathbf{1 0}}$ have presented a generalised framework for the analysis of base-excited rotor systems including large gyroscopic and Coriolis effects. Hori and Kato ${ }^{11}$ confined their study to investigate instability effects due to heavy shocks similar to those associated with strong earthquakes. Choy et al. ${ }^{12}$ investigated the non-linear behaviour of turbomachinery caused by rub events that take place under extreme operating conditions such as blade loss or seismic disturbances. Singh et al. ${ }^{13}$ examined the use of a modal ap- 